\title{
Robust Sliding Mode Predictive Control of Uncertain Networked Control System with Random Time Delay
}

\author{
Yu Zhang ${ }^{D},{ }^{1}$ Shousheng Xie, ${ }^{1}$ Ledi Zhang, ${ }^{1}$ and Litong Ren ${ }^{2}$ \\ ${ }^{1}$ Aeronautics and Astronautics Engineering Institute, Air Force Engineering University of PLA, Xian 710038, China \\ ${ }^{2}$ Unit 94314 of Chinese PLA, Zhengzhou 450003, China \\ Correspondence should be addressed to Yu Zhang; frank_sharon1314@126.com
}

Received 10 April 2018; Revised 21 June 2018; Accepted 9 July 2018; Published 19 July 2018

Academic Editor: Zhengqiu Zhang

Copyright (c) 2018 Yu Zhang et al. This is an open access article distributed under the Creative Commons Attribution License, which permits unrestricted use, distribution, and reproduction in any medium, provided the original work is properly cited.

\begin{abstract}
This paper proposes a sliding mode predictive controller with a new robust global sliding surface for a certain networked control system with random time delay, mismatched parametric uncertainty, and external disturbances. First, the model of the networked control system is established, based on which linear transformation is made to get a new form of the system which does not have time delay term in expression. Then a global sliding surface is proposed followed by the sufficient condition given in the form of linear matrix inequality (LMI) to guarantee system stability and robustness. Subsequently, a sliding mode predictive controller is proposed with modified reaching law as its reference trajectory and the rolling optimization method is combined to provide optimal control input for each step so that chattering can be minimized. Finally, simulations have been made and the results indicate the advantages of the proposed controller in the aspect of convergence speed, chattering suppression, and robustness to uncertainties.
\end{abstract}

\section{Introduction}

Because smart sensors and actuators with individual communication modules and processing units are adopted which allow for local control actions and communication is realized through a digital communication network with a more robust network such as a mesh, ring, or bus topology, networked control systems can overcome many disadvantages of the traditional control systems. However, the introduction of databus brings in together with it some inevitable problems such as time delay and packet dropout, which can damage system performance or even result in system instability. So it is of great importance to study the design of controller in presence of time delay. Great contributions have been made by researches on the study of networked control system with time delay [1-3]. In particular, [4] presents a novel fuzzy sliding mode controller and the networked-induced delay is handled by Pade approximation; [5] proposes a network time delay compensation method based on time delay prediction, where the predicted time delay is used instead of the actual time delay as the parameters of the network time delay compensation controller; and [6] designed an event-triggered predictor-based controller with sampled measurements where predictor models are chosen depending on the delay uncertainty. In addition, networked control systems also suffer from different kinds of uncertainties such as parameter perturbations, modeling errors, external disturbances, and other sort of uncertainties. As a result, a control strategy that takes into account main uncertainties and can handle nonlinearity with a certain robustness degree is required.

Sliding mode control (SMC) has recently attracted great attention owing to its attractive features such as fast response, good transient performance, and, most importantly, its insensitivity to uncertainties, which makes it fairly suitable for the nonlinear or uncertain systems [7-9]. Sliding mode control design is composed of two steps. In the first step, a sliding surface is designed, to which the plants dynamics is restricted during the sliding phase. In the second step, a control law is designed so that the system trajectory can converge to the sliding surface in finite time [10]. However, because of the existence of inertia and the limitation of control force, when system state reaches the designed sliding surface, it will not slide stably on the sliding surface as expected, but 
will move in a zigzag path along the sliding surface, which is known as chattering. If the degree of chattering cannot be controlled, it will damage system stability and deteriorate the performance of controller. Model-based predictive control (MPC) is a popular control methodology that has been successfully implemented in many industrial applications, showing good performance [11-13], and it is found recently that the introduction of MPC in SMC can effectively suppress chattering because the idea of multistep prediction and rolling optimization of MPC can optimize the reaching phase and obtain the optimal switching control signal of each step during the sliding phase [14-18].

In this paper, a sliding mode predictive controller has been proposed for a certain networked control system with random time delay, mismatched parametric uncertainty, and external disturbances. First, the networked control system model is established with clear definition of time delay and uncertainties condition and a linear transformation method is adopted to obtain a discrete system model which does not have time delay term in expression. Second, a robust global sliding surface is designed which has certain robustness to mismatched uncertainties and the sufficient condition for system stability and robustness on the sliding surface is given in the form of LMI. Third, a sliding mode predictive controller is proposed with a modified reaching law as its reference trajectory. Finally, simulations are made to test the effectiveness of the proposed controller and the results indicate that the proposed controller is advanced in the aspect of chattering suppression and robustness to uncertainties.

\section{System Model Description}

The simplified data communication scheme of the networked control system is shown in Figure 1.

It is assumed that sensors and actuators are time driven while the controller is event driven; data packet has time stamp and packets time disorder does not exist. It can be seen from Figure 1 that $\tau_{\mathrm{ca}}$ is controller-actuator time delay and $\tau_{\mathrm{sc}}$ is sensor-actuator time delay. According to [17], $\tau_{\mathrm{ca}}$ and $\tau_{\mathrm{sc}}$ can be combined as $\tau(k)$ to make it easier for analysis, based on which the discrete system model is established as follows:

$$
\begin{aligned}
\widehat{\boldsymbol{x}}(k+1) & =(\widehat{\boldsymbol{A}}+\Delta \widehat{\boldsymbol{A}}) \widehat{\boldsymbol{x}}(k)+\widehat{\boldsymbol{B}} \boldsymbol{u}(k-\tau(k))+\boldsymbol{R} \boldsymbol{d}(k) \\
\boldsymbol{u}(k) & =\boldsymbol{\chi}(k) \quad k \in\left[-\tau_{\max }, 0\right],
\end{aligned}
$$

where $\widehat{\boldsymbol{x}}(k) \in \mathrm{R}^{n}$ is system state variable; $\boldsymbol{u}(k) \in \mathrm{R}^{m}$ is control input; $\Delta \widehat{\boldsymbol{A}} \in \mathrm{R}^{n \times n}$ is mismatched model parameter perturbation; $\boldsymbol{d}(k) \in \mathrm{R}^{p}(p \leq n)$ is external disturbance; and $\widehat{\boldsymbol{A}}, \widehat{\boldsymbol{B}}, \boldsymbol{R}$ are matrices with appropriate dimensions.

Assumption 1. Matrix pair $(\widehat{\boldsymbol{A}}, \widehat{\boldsymbol{B}})$ is controllable and $\widehat{\boldsymbol{B}}$ is a matrix of full column rank, which satisfies $\operatorname{rank}(\widehat{\boldsymbol{B}})=m<n$; all system state variables are observable.

Assumption 2. Parameter perturbation matrix $\Delta \widehat{\boldsymbol{A}}$ satisfies

$$
\Delta \widehat{\boldsymbol{A}}=\boldsymbol{G} \boldsymbol{F}(k) \boldsymbol{H},
$$

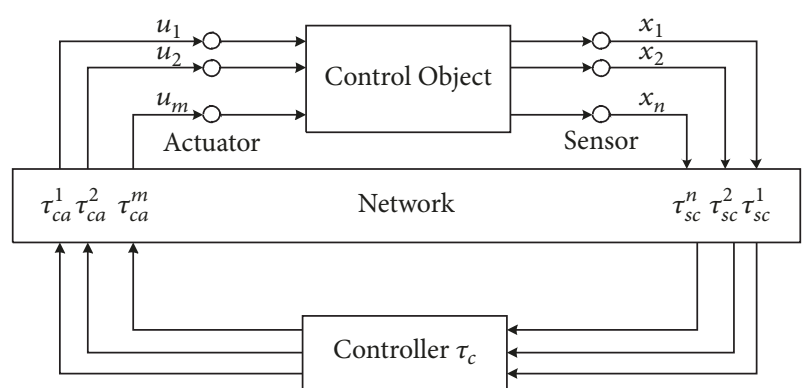

FIGURE 1: Data communication scheme of the networked control system.

where $\boldsymbol{F}(k)$ is unknown but bounded, which satisfies $\boldsymbol{F}^{\mathrm{T}}(k) \boldsymbol{F}(k) \leq \boldsymbol{I}$.

Assumption 3. External disturbance $\boldsymbol{d}(k)$ is bounded.

Time delay $\tau(k)$ is a bounded Markov random variable, whose state space is $\boldsymbol{\Omega}=\{1,2,3\}$ and the transition of time delay state can be described as

$$
\begin{gathered}
\tau(k+1)=\tau(k) \cdot \pi_{i j} \\
\sum_{j=1}^{3} \pi_{i j}=1
\end{gathered}
$$

$$
i, j \in \Omega
$$

with the time delay state transition matrix defined as $\Pi=\pi_{i j}$, $(i, j \in \boldsymbol{\Omega})$.

Because the existence of time delay term $\tau(k)$ in system (1) can bring a huge trouble to the controller design, a linear transformation method [19] is used here to get a new form of the system which does not have time delay term in expression. The linear transformation is defined as

$$
\boldsymbol{x}(k)=\overline{\boldsymbol{A}}^{\tau} \widehat{\boldsymbol{x}}(k)+\sum_{i=0}^{\tau-1} \overline{\boldsymbol{A}}^{\tau-i-1} \widehat{\boldsymbol{B}} \boldsymbol{u}(k-\tau+i),
$$

where $\overline{\boldsymbol{A}}=\widehat{\boldsymbol{A}}+\Delta \widehat{\boldsymbol{A}}$. Substitute (4) into system (1) yielding the equivalent system as follows:

$$
\boldsymbol{x}(k+1)=\overline{\boldsymbol{A}} \boldsymbol{x}(k)+\widehat{\boldsymbol{B}} \boldsymbol{u}(k)+\boldsymbol{Q} \boldsymbol{d}(k),
$$

where $\boldsymbol{Q} \boldsymbol{d}(k)=(\widehat{\boldsymbol{A}}+\Delta \widehat{\boldsymbol{A}})^{\tau} \boldsymbol{R} \boldsymbol{d}(k)$ and it is known from Assumption 1 that system (5) is controllable.

Proof. Decompose $\widehat{\boldsymbol{B}} \boldsymbol{u}(k-\tau)$ as follows:

$$
\begin{aligned}
\widehat{\boldsymbol{B}} \boldsymbol{u}(k-\tau)= & \widehat{\boldsymbol{B}} \boldsymbol{u}(k-\tau)-\overline{\boldsymbol{A}}^{-1} \widehat{\boldsymbol{B}} \boldsymbol{u}(k-\tau+1) \\
& +\overline{\boldsymbol{A}}^{-1} \widehat{\boldsymbol{B}} \boldsymbol{u}(k-\tau+1)-\cdots \\
& -\overline{\boldsymbol{A}}^{-\tau+1} \widehat{\boldsymbol{B}} \boldsymbol{u}(k-1)+\overline{\boldsymbol{A}}^{-\tau+1} \widehat{\boldsymbol{B}} \boldsymbol{u}(k-1) \\
& -\overline{\boldsymbol{A}}^{\tau} \widehat{\boldsymbol{B}} \boldsymbol{u}(k)+\overline{\boldsymbol{A}}^{-\tau} \widehat{\boldsymbol{B}} \boldsymbol{u}(k)
\end{aligned}
$$




$$
\begin{aligned}
= & \overline{\boldsymbol{A}}^{-\tau} \widehat{\boldsymbol{B}} \boldsymbol{u}(k)+\sum_{i=0}^{\tau-1} \overline{\boldsymbol{A}}^{-i} \widehat{\boldsymbol{B}} \boldsymbol{u}(k-\tau+i) \\
& -\sum_{i=0}^{\tau-1} \overline{\boldsymbol{A}}^{-i-1} \widehat{\boldsymbol{B}} \boldsymbol{u}(k-\tau+1+i)
\end{aligned}
$$

Substitute (6) into (1), yielding

$$
\begin{aligned}
\widehat{\boldsymbol{x}}(k+1)+\sum_{i=0}^{\tau-1} \overline{\boldsymbol{A}}^{-i-1} \widehat{\boldsymbol{B}} \boldsymbol{u}(k-\tau+1+i) \\
=\overline{\boldsymbol{A}} \widehat{\boldsymbol{x}}(k)+\overline{\boldsymbol{A}}^{-\tau} \widehat{\boldsymbol{B}} \boldsymbol{u}(k)+\sum_{i=0}^{\tau-1} \overline{\boldsymbol{A}}^{-i} \widehat{\boldsymbol{B}} \boldsymbol{u}(k-\tau+i) \\
+\boldsymbol{R} \boldsymbol{d}(k) \\
=\overline{\boldsymbol{A}}\left[\widehat{\boldsymbol{x}}(k)+\sum_{i=0}^{\tau-1} \overline{\boldsymbol{A}}^{-i-1} \widehat{\boldsymbol{B}} \boldsymbol{u}(k-\tau+i)\right]+\overline{\boldsymbol{A}}^{-\tau} \widehat{\boldsymbol{B}} \boldsymbol{u}(k) \\
+\boldsymbol{R} \boldsymbol{d}(k)
\end{aligned}
$$

Multiply both sides of (7) by $\overline{\boldsymbol{A}}^{\tau}$, yielding

$$
\begin{aligned}
\overline{\boldsymbol{A}}^{\tau} \widehat{\boldsymbol{x}}(k+1)+\sum_{i=0}^{\tau-1} \overline{\boldsymbol{A}}^{\tau-i-1} \widehat{\boldsymbol{B}} \boldsymbol{u}(k-\tau+1+i) \\
=\overline{\boldsymbol{A}}\left[\overline{\boldsymbol{A}}^{\tau} \widehat{\boldsymbol{x}}(k)+\sum_{i=0}^{\tau-1} \overline{\boldsymbol{A}}^{\tau-i-1} \widehat{\boldsymbol{B}} \boldsymbol{u}(k-\tau+i)\right]+\widehat{\boldsymbol{B}} \boldsymbol{u}(k) \\
+\overline{\boldsymbol{A}}^{\tau} \boldsymbol{R} \boldsymbol{d}(k)
\end{aligned}
$$

Taking $\overline{\boldsymbol{A}}^{\tau} \hat{\boldsymbol{x}}(k)+\sum_{i=0}^{\tau-1} \overline{\boldsymbol{A}}^{\tau-i-1} \widehat{\boldsymbol{B}} \boldsymbol{u}(k-\tau+i)$ as the new equivalent system state variable, (5) can be obtained.

\section{Sliding Mode Controller Design}

3.1. Sliding Mode Regular Form of System. First of all, the system is transformed into the sliding mode regular form so that the design of sliding mode controller can be carried out clearly in two parts, which are sliding mode motion and approaching motion.

Considering system (5), define a nonsingular matrix $\boldsymbol{T} \epsilon$ $\mathrm{R}^{n \times n}$, which makes

$$
\boldsymbol{T} \widehat{\boldsymbol{B}}=\left[\begin{array}{c}
0_{(n-m) \times m} \\
\boldsymbol{B}_{m}
\end{array}\right],
$$

where $\boldsymbol{B}_{m} \in \mathrm{R}^{m \times m}$ is nonsingular. For convenience, choose $\boldsymbol{T}=\left[\begin{array}{ll}\boldsymbol{U}_{2} & \boldsymbol{U}_{1}\end{array}\right]^{T}$, where $\boldsymbol{U}_{1} \in \mathrm{R}^{n \times m}, \boldsymbol{U}_{2} \in \mathrm{R}^{n \times(n-m)}$ are two subblocks of a unitary matrix resulting from the singular value decomposition of $\widehat{\boldsymbol{B}}$, i.e.,

$$
\widehat{\boldsymbol{B}}=\left[\begin{array}{ll}
\boldsymbol{U}_{1} & \boldsymbol{U}_{2}
\end{array}\right]\left[\begin{array}{c}
\boldsymbol{\Sigma}_{m \times m} \\
0_{(n-m) \times m}
\end{array}\right] \boldsymbol{V}^{\mathrm{T}},
$$

where $\boldsymbol{\Sigma}$ is a diagonal positive-definite matrix and $\boldsymbol{V}$ is usually a unitary matrix.

Define $\boldsymbol{z}=\boldsymbol{T} \boldsymbol{x}$; the regular form of system (5) is obtained as follows:

$$
\boldsymbol{z}(k+1)=(\boldsymbol{A}+\Delta \boldsymbol{A}) \boldsymbol{z}(k)+\boldsymbol{B} \boldsymbol{u}(k)+\boldsymbol{\omega}(k),
$$

where $\boldsymbol{B}=\left[\begin{array}{c}0_{(n-m) \times m} \\ \boldsymbol{B}_{m}\end{array}\right]=\boldsymbol{T} \widehat{\boldsymbol{B}}$.

Decomposing (11) yields the sliding mode motion (12a) and the approaching motion (12b) as follows:

$$
\begin{aligned}
z_{1}(k+1)= & \left(\boldsymbol{A}_{11}+\Delta \boldsymbol{A}_{11}\right) \boldsymbol{z}_{1}(k) \\
& +\left(\boldsymbol{A}_{12}+\Delta \boldsymbol{A}_{12}\right) \boldsymbol{z}_{2}(k)+\boldsymbol{\omega}_{1}(k), \\
\boldsymbol{z}_{2}(k+1)= & \left(\boldsymbol{A}_{21}+\Delta \boldsymbol{A}_{21}\right) \boldsymbol{z}_{1}(k) \\
& +\left(\boldsymbol{A}_{22}+\Delta \boldsymbol{A}_{22}\right) \boldsymbol{z}_{2}(k)+\boldsymbol{B}_{m} \boldsymbol{u}(k) \\
& +\boldsymbol{\omega}_{2}(k),
\end{aligned}
$$

where $\boldsymbol{z}_{1}(k) \in \mathrm{R}^{n-m}, \boldsymbol{z}_{2}(k) \in \mathrm{R}^{m} ; \boldsymbol{A}=\boldsymbol{T} \widehat{\boldsymbol{A}} \boldsymbol{T}^{-1}, \Delta \boldsymbol{A}=$ $\boldsymbol{T} \Delta \widehat{\boldsymbol{A}} \boldsymbol{T}^{-1}$, and it can be obtained from (12a) and (12b) that $\Delta \boldsymbol{A}_{11}=\boldsymbol{U}_{2}^{\mathrm{T}} \Delta \widehat{\boldsymbol{A}} \boldsymbol{U}_{2}, \Delta \boldsymbol{A}_{12}=\boldsymbol{U}_{2}^{\mathrm{T}} \Delta \widehat{\boldsymbol{A}} \boldsymbol{U}_{1}, \Delta \boldsymbol{A}_{21}=\boldsymbol{U}_{1}^{\mathrm{T}} \Delta \widehat{\boldsymbol{A}} \boldsymbol{U}_{2}$, $\Delta \boldsymbol{A}_{22}=\boldsymbol{U}_{1}^{\mathrm{T}} \Delta \widehat{\boldsymbol{A}} \boldsymbol{U}_{1}$. The disturbance term now becomes

$$
\boldsymbol{\omega}(k)=\boldsymbol{T}(\widehat{\boldsymbol{A}}+\Delta \widehat{\boldsymbol{A}})^{\tau} \boldsymbol{R} \boldsymbol{d}(k) .
$$

3.2. Robust Global Sliding Surface Design. Define the following global sliding surface:

$$
\begin{aligned}
\boldsymbol{s}(k) & =\boldsymbol{C} \boldsymbol{z}(k)-\beta^{k} \boldsymbol{C} \boldsymbol{z}(0) \\
& =\left[\begin{array}{ll}
\boldsymbol{K} & \boldsymbol{I}
\end{array}\right]\left[\begin{array}{ll}
\boldsymbol{z}_{1}^{\mathrm{T}}(k) & \boldsymbol{z}_{2}^{\mathrm{T}}(k)
\end{array}\right]^{\mathrm{T}}-\beta^{k} \boldsymbol{C} \boldsymbol{z}(0),
\end{aligned}
$$

where $0<\beta<1, \boldsymbol{z}(0)=\boldsymbol{T} \boldsymbol{x}(0)$ and $\boldsymbol{C}$ is the sliding surface parameter to be designed. Here it is necessary to figure out that $\boldsymbol{C}$ in the term $\beta^{k} \boldsymbol{C} \boldsymbol{z}(0)$ is not expressed as $\left[\begin{array}{ll}\boldsymbol{K} & \boldsymbol{I}\end{array}\right]$ because $\beta^{k} \boldsymbol{C z}(0)$ will be taken as a whole in the proceeding proof process. Let $\boldsymbol{s}(k)=0$, yielding

$$
\boldsymbol{z}_{2}(k)=-\boldsymbol{K} \boldsymbol{z}_{1}(k)+\beta^{k} \boldsymbol{C} \boldsymbol{z}(0) .
$$

Substitute (15) into (12a) and according to Assumption 2, we have

$$
\begin{aligned}
\boldsymbol{z}_{1}( & +1) \\
= & \left(\boldsymbol{A}_{11}+\Delta \boldsymbol{A}_{11}\right) \boldsymbol{z}_{1}(k)+\left(\boldsymbol{A}_{12}+\Delta \boldsymbol{A}_{12}\right) \\
& \cdot\left(-\boldsymbol{K} \boldsymbol{z}_{1}(k)+\beta^{k} \boldsymbol{C} \boldsymbol{z}(0)\right)+\boldsymbol{\omega}_{1}(k) \\
= & \left(\boldsymbol{A}_{11}-\boldsymbol{A}_{12} \boldsymbol{K}+\boldsymbol{U}_{2}^{\mathrm{T}} \Delta \widehat{\boldsymbol{A}} \boldsymbol{U}_{2}-\boldsymbol{U}_{2}^{\mathrm{T}} \Delta \widehat{\boldsymbol{A}} \boldsymbol{U}_{1} \boldsymbol{K}\right) \boldsymbol{z}_{1}(k) \\
& +\left(\boldsymbol{A}_{12}+\Delta \boldsymbol{A}_{12}\right) \beta^{k} \boldsymbol{C} \boldsymbol{z}(0)+\boldsymbol{\omega}_{1}(k) \\
= & {\left[\boldsymbol{A}_{11}-\boldsymbol{A}_{12} \boldsymbol{K}+\boldsymbol{U}_{2}^{\mathrm{T}} \boldsymbol{G} \boldsymbol{F}(k) \boldsymbol{H}\left(\boldsymbol{U}_{2}-\boldsymbol{U}_{1} \boldsymbol{K}\right)\right] \boldsymbol{z}_{1}(k) } \\
& +\left(\boldsymbol{A}_{12}+\boldsymbol{U}_{2}^{\mathrm{T}} \boldsymbol{G} \boldsymbol{F}(k) \boldsymbol{H} \boldsymbol{U}_{1}\right) \beta^{k} \boldsymbol{C} \boldsymbol{z}(0)+\boldsymbol{\omega}_{1}(k) .
\end{aligned}
$$


Lemma 4. Let $\boldsymbol{H}, \boldsymbol{G}$, and $\boldsymbol{F}$ be real matrices of appropriate dimensions with $\boldsymbol{F}$ satisfying $\boldsymbol{F}^{T} \boldsymbol{F} \leq \boldsymbol{I}$. Then for arbitrary scalar $\varepsilon>0$, we have

$$
\boldsymbol{H F G}+\boldsymbol{G}^{T} \boldsymbol{F}^{T} \boldsymbol{H}^{T} \leq \boldsymbol{\varepsilon} \boldsymbol{H} \boldsymbol{H}^{T}+\boldsymbol{\varepsilon}^{-1} \boldsymbol{G}^{T} \boldsymbol{G} .
$$

Theorem 5. For the given positive constant $\gamma$, the augmented sliding mode motion (16) is asymptotically stable on sliding surface (14) with a noise attenuation level $\gamma$ upon the external disturbance, if there exist symmetrical positive determined matrix $\boldsymbol{X}$, matrix $\boldsymbol{W}$, and scalar $\varepsilon>0$ satisfying the following linear matrix inequality (LMI):

$$
\left[\begin{array}{ccccc}
-\boldsymbol{X} & 0 & * & * & * \\
0 & -\gamma^{2} \boldsymbol{I} & * & * & * \\
\boldsymbol{\Xi}_{1} & \boldsymbol{I} & \boldsymbol{\Xi}_{2} & * & * \\
\boldsymbol{X} & 0 & 0 & -\boldsymbol{I} & * \\
\boldsymbol{\Xi}_{3} & 0 & 0 & 0 & -\varepsilon \boldsymbol{I}
\end{array}\right]<0
$$

where $\boldsymbol{\Xi}_{1}=\boldsymbol{A}_{11} \boldsymbol{X}-\boldsymbol{A}_{12} \boldsymbol{W}, \boldsymbol{\Xi}_{2}=-\boldsymbol{X}+\varepsilon \boldsymbol{U}_{2}^{\mathrm{T}} \boldsymbol{G}\left(\boldsymbol{U}_{2}^{\mathrm{T}} \boldsymbol{G}\right)^{\mathrm{T}}, \boldsymbol{\Xi}_{3}=$ $\left(\boldsymbol{H}_{\mathrm{A}} \boldsymbol{U}_{2}\right) \boldsymbol{X}-\left(\boldsymbol{H}_{\mathrm{A}} \boldsymbol{U}_{1}\right) \boldsymbol{W}$, and the sliding surface parameter is thus obtained as $\boldsymbol{K}=\boldsymbol{W} \boldsymbol{X}^{-1}$.

Proof: Define the Lyapunov function as

$$
\boldsymbol{V}\left(\boldsymbol{z}_{1}(k), k\right)=\boldsymbol{z}_{1}^{\mathrm{T}}(k) \boldsymbol{P} \boldsymbol{z}_{1}(k) .
$$

Along the trajectory of system (16), we have

$$
\begin{aligned}
\Delta & \boldsymbol{V}(k)=\boldsymbol{V}\left(\boldsymbol{z}_{1}(k+1), k+1\right)-\boldsymbol{V}\left(\boldsymbol{z}_{1}(k), k\right) \\
& =\boldsymbol{z}_{1}^{\mathrm{T}}(k) \\
& \cdot\left[\boldsymbol{A}_{11}-\boldsymbol{A}_{12} \boldsymbol{K}+\boldsymbol{U}_{2}^{\mathrm{T}} \boldsymbol{G} \boldsymbol{F}(k) \boldsymbol{H}\left(\boldsymbol{U}_{2}-\boldsymbol{U}_{1} \boldsymbol{K}\right)\right]^{\mathrm{T}} \boldsymbol{P} \\
& \cdot\left[\boldsymbol{A}_{11}-\boldsymbol{A}_{12} \boldsymbol{K}+\boldsymbol{U}_{2}^{\mathrm{T}} \boldsymbol{G} \boldsymbol{F}(k) \boldsymbol{H}\left(\boldsymbol{U}_{2}-\boldsymbol{U}_{1} \boldsymbol{K}\right)\right] \boldsymbol{z}_{1}(k) \\
& +\boldsymbol{\omega}_{1}^{\mathrm{T}}(k) \\
& \cdot \boldsymbol{P}\left[\boldsymbol{A}_{11}-\boldsymbol{A}_{12} \boldsymbol{K}+\boldsymbol{U}_{2}^{\mathrm{T}} \boldsymbol{G} \boldsymbol{F}(k) \boldsymbol{H}\left(\boldsymbol{U}_{2}-\boldsymbol{U}_{1} \boldsymbol{K}\right)\right] \\
& \cdot \boldsymbol{z}_{1}(k)+\boldsymbol{z}_{1}^{\mathrm{T}}(k) \\
& \cdot\left[\boldsymbol{A}_{11}-\boldsymbol{A}_{12} \boldsymbol{K}+\boldsymbol{U}_{2}^{\mathrm{T}} \boldsymbol{G} \boldsymbol{F}(k) \boldsymbol{H}\left(\boldsymbol{U}_{2}-\boldsymbol{U}_{1} \boldsymbol{K}\right)\right]^{\mathrm{T}} \\
& \cdot \boldsymbol{P} \boldsymbol{\omega}_{1}(k)+\boldsymbol{\omega}_{1}^{\mathrm{T}}(k) \boldsymbol{P} \boldsymbol{\omega}_{1}(k) \\
& +\left[\beta^{k}\left(\boldsymbol{A}_{12}+\boldsymbol{U}_{2}^{\mathrm{T}} \boldsymbol{G} \boldsymbol{F}(k) \boldsymbol{H} \boldsymbol{U}_{1}\right) \boldsymbol{C} \boldsymbol{z}(0)\right]^{\mathrm{T}} \boldsymbol{P} \\
& \cdot\left[\beta^{k}\left(\boldsymbol{A}_{12}+\boldsymbol{U}_{2}^{\mathrm{T}} \boldsymbol{G} \boldsymbol{F}(k) \boldsymbol{H} \boldsymbol{U}_{1}\right) \boldsymbol{C} \boldsymbol{z}(0)\right]-\boldsymbol{z}_{1}^{\mathrm{T}}(k) \\
& \cdot P \boldsymbol{z}_{1}(k) .
\end{aligned}
$$

Define $\boldsymbol{\xi}(k)=\left[\begin{array}{ll}\boldsymbol{z}_{1}(k) & \boldsymbol{\omega}_{1}(k)\end{array}\right]^{\mathrm{T}}$, then we get

$$
\begin{aligned}
& \Delta \boldsymbol{V}(k)=\boldsymbol{\xi}^{\mathrm{T}}(k)\left\{\left[\left(\boldsymbol{A}_{11}-\boldsymbol{A}_{12} \boldsymbol{K}+\Theta\right) \boldsymbol{I}\right]^{T}\right. \\
& \cdot \boldsymbol{P}\left[\left(\boldsymbol{A}_{11}-\boldsymbol{A}_{12} \boldsymbol{K}+\Theta\right) \boldsymbol{I}\right]+\left[\begin{array}{cc}
-\boldsymbol{P} & 0 \\
0 & -\gamma^{2} \boldsymbol{I}
\end{array}\right] \\
& \left.\quad+\left[\begin{array}{ll}
\boldsymbol{I} & 0 \\
0 & 0
\end{array}\right]\right\} \boldsymbol{\xi}(k)+\gamma^{2} \boldsymbol{\omega}_{1}^{\mathrm{T}}(k) \boldsymbol{\omega}_{1}(k)+\left(\beta^{k} \boldsymbol{\Pi} \boldsymbol{C} \boldsymbol{z}(0)\right)^{T} \\
& \quad \cdot \boldsymbol{P}\left(\beta^{k} \boldsymbol{\Pi} \boldsymbol{C} \boldsymbol{z}(0)\right)-\boldsymbol{z}_{1}^{\mathrm{T}}(k) \boldsymbol{z}_{1}(k),
\end{aligned}
$$

where $\boldsymbol{\Theta}=\boldsymbol{U}_{2}^{\mathrm{T}} \boldsymbol{G} \boldsymbol{F}(k) \boldsymbol{H}_{\mathrm{A}}\left(\boldsymbol{U}_{2}-\boldsymbol{U}_{1} \boldsymbol{K}\right), \boldsymbol{\Pi}=\boldsymbol{A}_{12}+$ $\boldsymbol{U}_{2}^{\mathrm{T}} \boldsymbol{G} \boldsymbol{F}(k) \boldsymbol{H}_{\mathrm{A}} \boldsymbol{U}_{1}$.

Since $\left(\beta^{k} \boldsymbol{\Pi} \boldsymbol{C z}(0)\right)^{T} \boldsymbol{P}\left(\beta^{k} \boldsymbol{\Pi} \boldsymbol{C} \boldsymbol{z}(0)\right)>0$, we have

$$
\begin{aligned}
& \Delta \boldsymbol{V}(k)<\boldsymbol{\xi}^{\mathrm{T}}(k)\left\{\left[\left(\boldsymbol{A}_{11}-\boldsymbol{A}_{12} \boldsymbol{K}+\Theta\right) \boldsymbol{I}\right]^{T}\right. \\
& \cdot \boldsymbol{P}\left[\left(\boldsymbol{A}_{11}-\boldsymbol{A}_{12} \boldsymbol{K}+\Theta\right) \boldsymbol{I}\right]+\left[\begin{array}{cc}
-\boldsymbol{P} & 0 \\
0 & -\gamma^{2} \boldsymbol{I}
\end{array}\right] \\
& \left.\quad+\left[\begin{array}{ll}
\boldsymbol{I} & 0 \\
0 & 0
\end{array}\right]\right\} \boldsymbol{\xi}(k)-\boldsymbol{z}_{1}^{\mathrm{T}}(k) \boldsymbol{z}_{1}(k)+\gamma^{2} \boldsymbol{\omega}_{1}^{\mathrm{T}}(k) \boldsymbol{\omega}_{1}(k) .
\end{aligned}
$$

It is obvious that when the following inequality holds,

$$
\begin{aligned}
& {\left[\left(\boldsymbol{A}_{11}-\boldsymbol{A}_{12} \boldsymbol{K}+\boldsymbol{\Theta}\right) \boldsymbol{I}\right]^{\mathrm{T}} \boldsymbol{P}\left[\left(\boldsymbol{A}_{11}-\boldsymbol{A}_{12} \boldsymbol{K}+\boldsymbol{\Theta}\right) \boldsymbol{I}\right]} \\
& \quad+\left[\begin{array}{cc}
-\boldsymbol{P} & 0 \\
0 & -\boldsymbol{\gamma}^{2} \boldsymbol{I}
\end{array}\right]+\left[\begin{array}{ll}
\boldsymbol{I} & 0 \\
0 & 0
\end{array}\right]<0,
\end{aligned}
$$

then we can obtain, according to (21) and (23), that

$$
\boldsymbol{z}_{1}^{\mathrm{T}}(k) \boldsymbol{z}_{1}(k)-\gamma^{2} \boldsymbol{\omega}_{1}^{\mathrm{T}}(k) \boldsymbol{\omega}_{1}(k)<-\Delta \boldsymbol{V}(k) .
$$

Summing both sides of (24) from $k=0$ to $\infty$ yields

$$
\sum_{k=0}^{\infty}\left[z_{1}^{\mathrm{T}}(k) z_{1}(k)-\gamma^{2} \boldsymbol{\omega}_{1}^{\mathrm{T}}(k) \boldsymbol{\omega}_{1}(k)\right]<-\boldsymbol{V}(\infty)<0 .
$$

Then it is obtained that $\sum_{k=0}^{\infty} z_{1}^{\mathrm{T}}(k) z_{1}(k)<$ $\gamma^{2} \sum_{k=0}^{\infty} \omega_{1}^{\mathrm{T}}(k) \boldsymbol{\omega}_{1}(k)$, which means sliding motion (16) is asymptotically stable with a noise attenuation level $\gamma$ upon the external disturbance $\omega_{1}(k)$.

According to Schur's complement, (23) is equivalent to

$$
\begin{aligned}
& \boldsymbol{M} \\
& =\left[\begin{array}{cccc}
-\boldsymbol{P} & 0 & \left(\boldsymbol{A}_{11}-\boldsymbol{A}_{12} \boldsymbol{K}+\boldsymbol{\Theta}\right)^{\mathrm{T}} & \boldsymbol{I} \\
0 & -\gamma^{2} \boldsymbol{I} & \boldsymbol{I} & 0 \\
\left(\boldsymbol{A}_{11}-\boldsymbol{A}_{12} \boldsymbol{K}+\boldsymbol{\Theta}\right) & \boldsymbol{I} & -\boldsymbol{P}^{-1} & 0 \\
\boldsymbol{I} & 0 & 0 & -\boldsymbol{I}
\end{array}\right] \\
& <0 .
\end{aligned}
$$


According to the definition of $\Theta$, (26) can be further decomposed as

$$
\begin{aligned}
& \boldsymbol{M}=\left[\begin{array}{cccc}
-\boldsymbol{P} & 0 & \left(\boldsymbol{A}_{11}-\boldsymbol{A}_{12} \boldsymbol{K}\right)^{\mathrm{T}} & \boldsymbol{I} \\
0 & -\gamma^{2} \boldsymbol{I} & \boldsymbol{I} & 0 \\
\left(\boldsymbol{A}_{11}-\boldsymbol{A}_{12} \boldsymbol{K}\right) & \boldsymbol{I} & -\boldsymbol{P}^{-1} & 0 \\
\boldsymbol{I} & 0 & 0 & -\boldsymbol{I}
\end{array}\right] \\
& +\left[\begin{array}{c}
0 \\
0 \\
\boldsymbol{U}_{2}^{\mathrm{T}} \boldsymbol{G} \\
0
\end{array}\right] \boldsymbol{F}(k)\left[\begin{array}{c}
\boldsymbol{H}\left(\boldsymbol{U}_{2}-\boldsymbol{U}_{1} \boldsymbol{K}\right)^{\mathrm{T}} \\
0 \\
0 \\
0
\end{array}\right]^{\mathrm{T}} \\
& +\left[\begin{array}{c}
\boldsymbol{H}\left(\boldsymbol{U}_{2}-\boldsymbol{U}_{1} \boldsymbol{K}\right)^{T} \\
0 \\
0 \\
0
\end{array}\right] \boldsymbol{F}^{\mathrm{T}}(k)\left[\begin{array}{c}
0 \\
0 \\
\boldsymbol{U}_{2}^{\mathrm{T}} \boldsymbol{G} \\
0
\end{array}\right]^{T}<0 .
\end{aligned}
$$

Left and right multiply matrix $\operatorname{diag}\left(\boldsymbol{P}^{-1}, \boldsymbol{I}, \boldsymbol{I}, \boldsymbol{I}, \boldsymbol{I}\right)$ and let $\boldsymbol{X}=\boldsymbol{P}^{-1}, \boldsymbol{W}=\boldsymbol{K} \boldsymbol{X}$, yielding (18).

3.3. Sliding Mode Predictive Controller Design. First of all, the nominal model of system (1) is taken as the prediction model, which is

$$
\boldsymbol{z}(k+1)=\boldsymbol{A} \boldsymbol{z}(k)+\boldsymbol{B} \boldsymbol{u}(k) .
$$

Then the sliding mode value of time $k+p$ is calculated as

$$
\begin{aligned}
\boldsymbol{s}_{y}(k+p)= & \boldsymbol{C} \boldsymbol{z}(k+p)-\beta^{k+p} \boldsymbol{C} \boldsymbol{z}(0) \\
= & \boldsymbol{C} \boldsymbol{A}^{p} \boldsymbol{z}(k)+\sum_{i=1}^{p} \boldsymbol{C} \boldsymbol{A}^{i-1} \boldsymbol{B} \boldsymbol{u}(k+p-i) \\
& -\beta^{k+p} \boldsymbol{C} \boldsymbol{z}(0) .
\end{aligned}
$$

Therefore, the predicted sliding mode value of time $k$ on time $k-p$ can be obtained as

$$
\begin{aligned}
\boldsymbol{s}_{y}(k / k-p)= & \boldsymbol{C} A^{p} \boldsymbol{z}(k-p)+\sum_{i=1}^{p} \boldsymbol{C} A^{i-1} \boldsymbol{B} \boldsymbol{u}(k-i) \\
& -\beta^{k} \boldsymbol{C} \boldsymbol{z}(0)
\end{aligned}
$$

Equation (30) can be described in a vector form:

$$
S_{y}(k+1)=\Phi Z(k)+\Psi U(k)+\Gamma(k)
$$

where

$$
\boldsymbol{S}_{\boldsymbol{y}}(k+1)=\left[s_{\boldsymbol{y}}(k+1), s_{\boldsymbol{y}}(k+2), \ldots, s_{\boldsymbol{y}}(k+N)\right]^{T},
$$

$$
\begin{aligned}
& \boldsymbol{Z}(k)=\left[\begin{array}{c}
\boldsymbol{z}(k) \\
\boldsymbol{z}(k) \\
\cdots \\
\boldsymbol{z}(k)
\end{array}\right]_{N \times 1}, \\
& \boldsymbol{\Phi}=\left[\begin{array}{llll}
(\boldsymbol{C A})^{T} & \left(\boldsymbol{C A}^{2}\right)^{T} & \cdots & \left(\boldsymbol{C A}^{N}\right)^{T}
\end{array}\right]^{T}, \\
& \boldsymbol{\Psi}=\left[\begin{array}{ccccc}
\boldsymbol{C B} & 0 & \cdots & \cdots & 0 \\
\boldsymbol{C A B} & \boldsymbol{C B} & \cdots & \cdots & 0 \\
\cdots & \cdots & \cdots & \cdots & \cdots \\
\boldsymbol{C} \boldsymbol{A}^{M-1} \boldsymbol{B} & \cdots & \cdots & \boldsymbol{C A B} & \boldsymbol{C B} \\
\cdots & \cdots & \cdots & \cdots & \cdots \\
\boldsymbol{C A}^{N-2} \boldsymbol{B} & \cdots & \cdots & \boldsymbol{C A}^{N-M} \boldsymbol{B} & \boldsymbol{C A}^{N-M-1} \boldsymbol{B} \\
\boldsymbol{C A}^{N-1} \boldsymbol{B} & \cdots & \cdots & \boldsymbol{C A}^{N-M+1} \boldsymbol{B} & \boldsymbol{C A}^{N-M} \boldsymbol{B}
\end{array}\right] \\
& \boldsymbol{U}(k)=[\boldsymbol{u}(k), \boldsymbol{u}(k+1), \ldots, \boldsymbol{u}(k+M-1)]^{T}, \\
& \Gamma(k)
\end{aligned}
$$

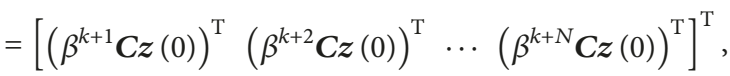

with $N$ being the prediction horizon and $M$ being the control horizon.

Then, a modified reaching law is chosen to be the reference trajectory of the control strategy. The concept of reaching law was first put forward in [20], and the corresponding discrete form of the reaching law is expressed as follows:

$$
\boldsymbol{s}(k+1)=(1-q T) \boldsymbol{s}(k)-\varepsilon T \operatorname{sgn}(\boldsymbol{s}(k)),
$$

where $\varepsilon>0, q>0,1-q T>0$, and $T$ is sampling period. However, for discrete systems, two critical problems limit the use of reaching law (33), which are as follows: (1) system state cannot be driven exactly to the origin; (2) the limitation of control force switching is not considered, which means when $s(k)$ is large, the switching of control force is too fierce to guarantee the stability of system state.

To overcome the imperfections of the traditional reaching law, the following reaching law is adopted [21, 22]:

$$
\begin{aligned}
\boldsymbol{s}(k+1)= & (1-q T) \boldsymbol{s}(k) \xi(\boldsymbol{s}(k)) \\
& -\varepsilon T \varphi(\boldsymbol{s}(k)) \operatorname{sgn}(\boldsymbol{s}(k)),
\end{aligned}
$$

where

$$
\begin{gathered}
\xi(s(k))= \begin{cases}1, & |s(k)|>\eta \\
0, & |s(k)| \leq \eta,\end{cases} \\
\varphi(s(k))= \begin{cases}1, & |s(k)|>\eta \\
\frac{|s(k)|^{2}}{\eta}, & |s(k)| \leq \eta,\end{cases}
\end{gathered}
$$

with $0<1-q T<1,0<\varepsilon T<1$, and $\eta=\varepsilon T /(1-q T)$. 
Theorem 6. With the sliding surface parameter $\boldsymbol{K}$ obtained in Theorem 5 and the global sliding surface given by (14), the trajectory of closed-loop system (16) can be driven onto the sliding surface through reaching law (34) and finally converges into a residual set of the origin $\{\boldsymbol{z}|| \boldsymbol{s}(k) \mid<\eta\}$.

Proof.

(1) If $|\boldsymbol{s}(k)|>\eta$, there is

$$
\boldsymbol{s}(k+1)=(1-q T) \boldsymbol{s}(k)-\varepsilon T \operatorname{sgn}(\boldsymbol{s}(k)) .
$$

Equation (37) is just the reaching law given in [20], so it is clear that the trajectory can enter the quasi-sliding mode area $\{\boldsymbol{z}|| \boldsymbol{s}(k) \mid<\eta\}$ in finite time.

(2) If $|\boldsymbol{s}(k)|<\eta$, we have $\boldsymbol{s}(k+1)=-(1-$ $q T)|\boldsymbol{s}(k)|^{2} \operatorname{sgn}(\boldsymbol{s}(k))$. Then the following two conditions are discussed.

(1) If $|\boldsymbol{s}(k)|=0$, there is $\boldsymbol{s}(k+1)=0$. Then it is clear that the trajectory is already stable on the sliding surface.

(2) If $|\boldsymbol{s}(k)| \neq 0$, choose a Lyapunov function as $\boldsymbol{V}(k)=\boldsymbol{s}^{2}(k)$.

Then we have

$$
\begin{aligned}
\Delta \boldsymbol{v}(k) & =\boldsymbol{v}(k+1)-\boldsymbol{v}(k) \\
& =\left[-(1-q T)|\boldsymbol{s}(k)|^{2} \operatorname{sgn}(\boldsymbol{s}(k))\right]^{2}-\boldsymbol{s}^{2}(k) \\
& =(1-q T)|\boldsymbol{s}(k)|^{4}-\boldsymbol{s}^{2}(k) \\
& =\boldsymbol{s}^{2}(k)\left[(1-q T)^{2} \boldsymbol{s}^{2}(k)-1\right] \\
& \leq \boldsymbol{s}^{2}(k)\left[\varepsilon^{2} T^{2}-1\right]<0 .
\end{aligned}
$$

Therefore, the system state will reach the sliding surface and remain there for the rest of time. is

According to (34), the reference sliding mode trajectory

$$
\begin{aligned}
\boldsymbol{s}_{\boldsymbol{r}}(k+p)= & (1-q T) \boldsymbol{s}_{\boldsymbol{r}}(k+p-1) \xi(\boldsymbol{s}(k)) \xi(s(k)) \\
& -\varepsilon T \operatorname{sgn}(\boldsymbol{s}(k+p-1)) \varphi(\boldsymbol{s}(k)) \\
\boldsymbol{s}_{\boldsymbol{r}}(k)= & \boldsymbol{s}(k) .
\end{aligned}
$$

Then, to overcome the impact of uncertainties, the error between the actual sliding mode value $s(k)$ and the predictive sliding mode value $\boldsymbol{s}_{y}(k / k-p)$ is utilized to offer feedback compensation for the prediction of future sliding mode value $\boldsymbol{s}_{\boldsymbol{y}}(k+p)$.

Define $\widehat{\boldsymbol{s}}_{y}(k+p)$ as the predicted value of the sliding mode value of time $k+p$ after feedback compensation is introduced. Thus we have

$$
\begin{aligned}
\widehat{\boldsymbol{s}}_{\boldsymbol{y}}(k+p)= & \boldsymbol{s}_{\boldsymbol{y}}(k+p)+\boldsymbol{h}_{\boldsymbol{p}} \boldsymbol{e}(k) \\
= & \boldsymbol{C} \boldsymbol{A}^{p} \boldsymbol{z}(k)+\sum_{i=1}^{p} \boldsymbol{C} \boldsymbol{A}^{i-1} \boldsymbol{B} \boldsymbol{u}(k+p-i) \\
& -\beta^{k+p} \boldsymbol{C} \boldsymbol{z}(0)+\boldsymbol{h}_{\boldsymbol{p}} \boldsymbol{e}(k),
\end{aligned}
$$

where $\boldsymbol{e}(k)=\boldsymbol{s}(k)-\boldsymbol{s}_{y}(k / k-p)$ is prediction error and $\boldsymbol{h}_{\boldsymbol{p}}$ is correction coefficient.

Then the vector form of (40) can be written as

$$
\widehat{\boldsymbol{S}}_{\boldsymbol{y}}(k+1)=\boldsymbol{S}_{\boldsymbol{y}}(k+1)+\boldsymbol{H}_{\boldsymbol{p}} \boldsymbol{E}(k),
$$

where

$$
\begin{aligned}
& \widehat{\boldsymbol{S}}_{\boldsymbol{y}}(k+1) \\
& \quad=\left[\widehat{\boldsymbol{s}}_{\boldsymbol{y}}(k+1), \widehat{\boldsymbol{s}}_{\boldsymbol{y}}(k+2), \ldots, \widehat{\boldsymbol{s}}_{\boldsymbol{y}}(k+N)\right]^{T},
\end{aligned}
$$

$\boldsymbol{E}(k)$

$$
\begin{aligned}
& \quad=\left[\boldsymbol{s}(k)-\boldsymbol{s}_{\boldsymbol{y}}(k / k-1), \cdots, \boldsymbol{s}(k)-\boldsymbol{s}_{\boldsymbol{y}}(k / k-N)\right]^{T}, \\
& \boldsymbol{H}_{\boldsymbol{p}}=\operatorname{diag}\left\{h_{p 1}, h_{p 2}, \ldots, h_{p n}\right\} .
\end{aligned}
$$

Normally, we set $h_{p 1}=1$ and $0<h_{p n}<\cdots<h_{p 2}<$ 1 , which means the feedback compensation is reduced as prediction proceeds.

At last, rolling optimization is introduced because the objective of predictive control is to determine a sequence of future control signals by optimizing a certain performance index, which is usually known as cost function. Such performance index is not a globally invariant function but a rolling updated performance index which is usually a minimized value in a limited time horizon.

The optimization cost function is defined as [21]

$$
\begin{aligned}
j_{p}= & \sum_{i=1}^{N} q_{i}\left[\widehat{\boldsymbol{s}}_{\boldsymbol{y}}(k+i)-\boldsymbol{s}_{\boldsymbol{r}}(k+i)\right]^{2} \\
& +\sum_{j=1}^{M} r_{i}[\boldsymbol{u}(k+j-1)]^{2},
\end{aligned}
$$

where $q_{i}$ is a nonnegative weight coefficient which decides the weight of errors of different sampling points in the performance index $j_{p}$ and $r_{i}$ is a positive weight coefficient which is used to constrain the control variables.

Equation (43) can be rewritten in the vector form:

$$
\begin{aligned}
\boldsymbol{J}_{\boldsymbol{p}} & =\left[\widehat{\boldsymbol{S}}_{\boldsymbol{y}}(k+1)-\boldsymbol{S}_{\boldsymbol{r}}(k+1)\right]^{T} \boldsymbol{Q}\left[\widehat{\boldsymbol{S}}_{\boldsymbol{y}}(k+1)\right. \\
& \left.-\boldsymbol{S}_{\boldsymbol{r}}(k+1)\right]+\boldsymbol{U}(k)^{T} \boldsymbol{R} \boldsymbol{U}(k)=[\boldsymbol{\Phi} \boldsymbol{Z}(k) \\
& \left.+\boldsymbol{\Psi} \boldsymbol{U}(k)+\boldsymbol{\Gamma}(k)+\boldsymbol{H}_{\boldsymbol{p}} \boldsymbol{E}(k)-\boldsymbol{S}_{\boldsymbol{r}}(k+1)\right]^{T} \boldsymbol{Q} \\
& \cdot\left[\boldsymbol{\Phi} \boldsymbol{Z}(k)+\boldsymbol{\Psi} \boldsymbol{U}(k)+\boldsymbol{\Gamma}(k)+\boldsymbol{H}_{\boldsymbol{p}} \boldsymbol{E}(k)\right. \\
& \left.-\boldsymbol{S}_{\boldsymbol{r}}(k+1)\right]+\boldsymbol{U}(k)^{T} \boldsymbol{R} \boldsymbol{U}(k),
\end{aligned}
$$

where $\boldsymbol{Q}=\operatorname{diag}\left[q_{1}, q_{2}, \cdots q_{N}\right], \boldsymbol{R}=\operatorname{diag}\left[r_{1}, r_{2}, \cdots r_{N}\right]$.

Let $\partial \boldsymbol{J}_{\boldsymbol{p}} / \partial \boldsymbol{U}(k)=0$, yielding the predictive sliding mode control law:

$$
\begin{aligned}
& \boldsymbol{U}(k)=-\left(\boldsymbol{\Psi}^{T} \mathbf{Q} \boldsymbol{\Psi}+\boldsymbol{R}\right)^{-1} \\
& \quad \cdot \boldsymbol{\Psi}^{T} \boldsymbol{Q}\left[\boldsymbol{\Phi} \boldsymbol{Z}(k)+\boldsymbol{\Gamma}(k)+\boldsymbol{H}_{\boldsymbol{p}} \boldsymbol{E}(k)-\boldsymbol{S}_{\boldsymbol{r}}(k+1)\right] .
\end{aligned}
$$


Then the system input at time $k$ is the first element of $\boldsymbol{U}(k)$, that is,

$$
\boldsymbol{u}(k)=g \boldsymbol{U}(k),
$$

where $g=[1,0, \cdots, 0]_{1 \times \mathrm{N}}$.

\section{Numerical Examples}

Consider the uncertain system given in (1), whose parameters are as follows:

$$
\begin{aligned}
& \widehat{\boldsymbol{A}}=\left[\begin{array}{ccc}
-0.8641 & 0.1491 & -0.01559 \\
-0.0073 & 0.9445 & -0.00532 \\
0.4759 & -0.08775 & 0.5990
\end{array}\right], \\
& \widehat{\boldsymbol{B}}=\left[\begin{array}{ll}
0.01935 & 0.00468 \\
0.01731 & 0.01059 \\
0.1853 & -0.0959
\end{array}\right], \\
& \boldsymbol{R}=\left[\begin{array}{lll}
0.2 & 0.1 & 0.3
\end{array}\right]^{\mathrm{T}}, \\
& \boldsymbol{G}=\operatorname{diag}(1,1,1),
\end{aligned}
$$

$$
\boldsymbol{H}=\left[\begin{array}{ccc}
0.2 & 0.1 & 0.18 \\
0.1 & 0.3 & 0.2 \\
0.12 & 0.21 & 0.1
\end{array}\right]
$$

The system sampling period is $T=20 \mathrm{~ms}$.

The $H_{\infty}$ performance index is taken as $\gamma=6$, and then Theorem 5 is solved through LMI toolbox, obtaining

$$
\begin{aligned}
\boldsymbol{X}^{*} & =0.1156, \\
\boldsymbol{W}^{*} & =\left[\begin{array}{c}
-0.0491 \\
0.0323
\end{array}\right], \\
\mathcal{\varepsilon}^{*} & =0.138
\end{aligned}
$$
$\left[\begin{array}{c}-0.4244 \\ 0.2795\end{array}\right]$.

Thus the sliding surface parameter can be obtained as $\boldsymbol{K}=$

The predictive sliding mode control parameters are defined as follows:

(1) The predictive horizon $N=8$ and the control horizon $M=3$.

(2) According to the dimension of sliding surface $\boldsymbol{s}(k)$, the correction coefficient matrix $H_{p}$ is defined as a diagonal matrix whose dimension is $2 \mathrm{~N}$ :

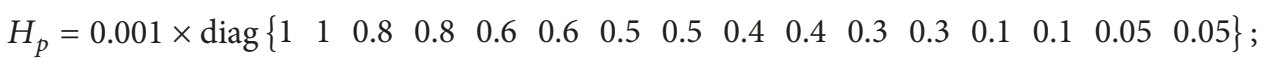

(3) The control weight coefficient is $G=0.001 \boldsymbol{I}_{2 M \times 2 M}$.

(4) The weight coefficient matrices $Q$ and $R$ are chosen as $Q=\boldsymbol{I}_{2 N \times 2 N}$ and $R=0.001 \boldsymbol{I}_{2 M \times 2 M}$.

To illustrate the performance of the proposed predictive sliding mode controller, a reaching law based sliding mode controller is introduced as comparison whose reaching law is (34) and it shares the same robust sliding surface with the proposed controller. Then the controller is designed as

$$
\begin{aligned}
\boldsymbol{u}(k) & =(\boldsymbol{C B})^{-1}[(1-q T) \boldsymbol{s}(k) \xi(\boldsymbol{s}(k)) \\
& \left.-\varepsilon T \varphi(\boldsymbol{s}(k)) \operatorname{sgn}(\boldsymbol{s}(k))+\beta^{k+1} \boldsymbol{C} \boldsymbol{z}(0)-\boldsymbol{C A} \boldsymbol{z}(k)\right] .
\end{aligned}
$$

The sliding surface parameter and the control law parameters for both controllers are the same, which are $\boldsymbol{C}=$ $\left[\begin{array}{ll}\boldsymbol{K} & \boldsymbol{I}_{2 \times 2}\end{array}\right]$ and $q=10, \varepsilon=0.5$. To simplify the expression, the proposed controller is denoted as RSMPC and the reaching law based controller is denoted as RSMC.

Time delay state transient matrix is defined as $\Pi=$ $\left[\begin{array}{lll}0.4 & 0.4 & 0.2 \\ 0.3 & 0.5 & 0.2 \\ 0.5 & 0.1 & 0.4\end{array}\right]$ and the according system time delay distribution is shown in Figure 2.

The initial system state is $\boldsymbol{x}(0)=\left[\begin{array}{lll}2 & 2 & 2\end{array}\right]^{T}$ and the external disturbance is defined as

$$
\boldsymbol{d}(k)=\boldsymbol{x}^{T}(k) \boldsymbol{N} \zeta(k)
$$

where $\zeta(k) \in \mathrm{R}^{3}$ is white Gaussian noise whose mean value is zero; $\boldsymbol{N}=\operatorname{diag}\left\{N_{1}, N_{2}, N_{3}\right\}$ is the upper bound of the noise intensity. Here the noise intensity is defined as $\boldsymbol{N}=$ $\operatorname{diag}\{0.05,0.05,0.05\}$. System state response curves with the two controllers are shown in Figure 3. It can be obtained from Figure 3 that, under the given time delay and disturbance conditions, both controllers can realize the finite time convergence of system states and because the reaching law of RSMC is the same as that used for the reference trajectory of the proposed controller, two controllers' dynamic performances are nearly the same. However, there is an obvious difference in the static performance of the two controllers. When RSMC is applied, system states suffer fierce chattering after reaching the neighborhood of the origin, which does not exist when the proposed controller is applied.

The evolutions of sliding surfaces and control inputs are shown in Figures 4 and 5, respectively. It can be seen that two controllers have similar dynamic performance but compared with the proposed controller, RSMC suffers much greater chattering both for sliding surface and for control inputs. The reasons for the above results can be explained as follows: the sliding surface parameter and the reaching law parameters of the two controllers are the same, which leads to the similarity of their dynamic performance. When the disturbances are in a relatively low level, both controllers are able to drive the system trajectory onto the sliding surface in a short time. 


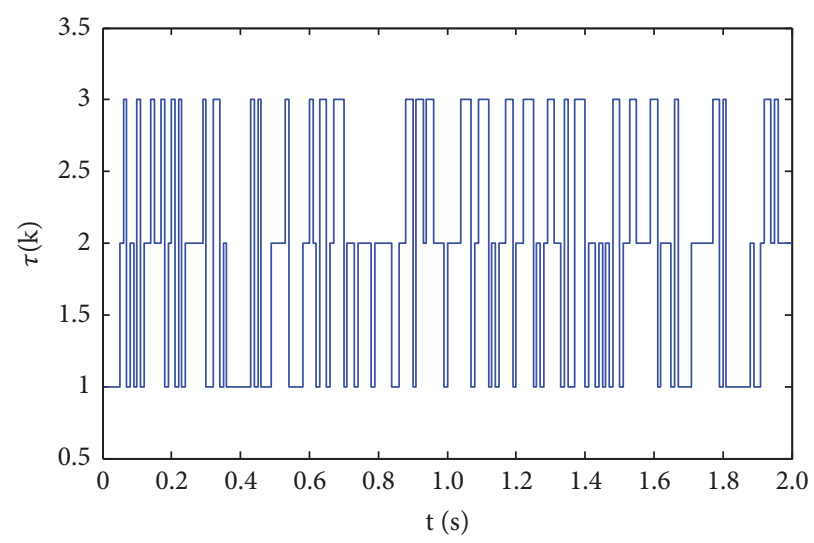

FIgURE 2: Distribution of system time delay.

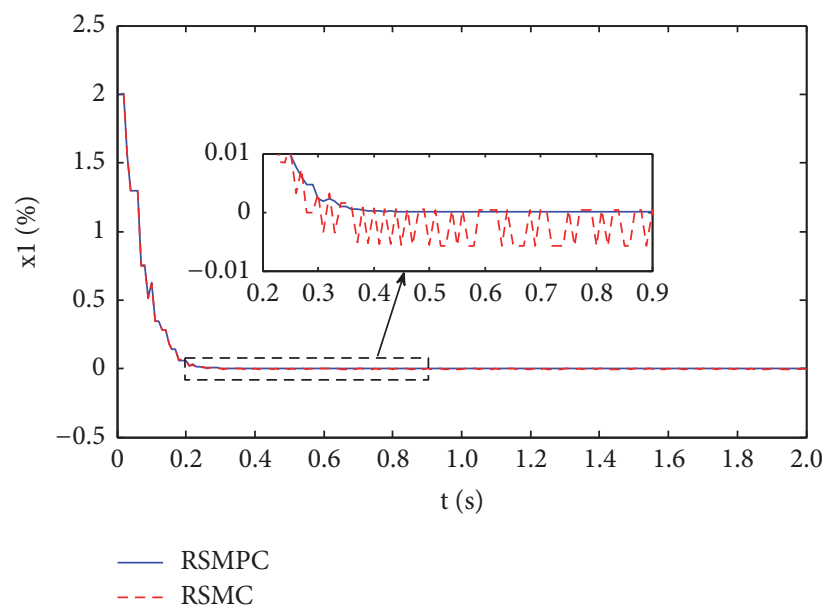

(a) Response curves of system state $x_{1}$

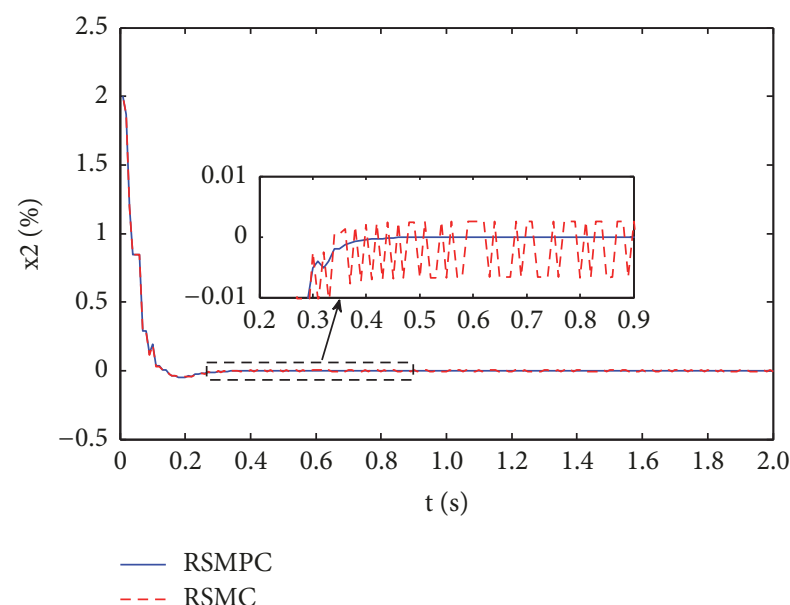

(b) Response curves of system state $x_{2}$

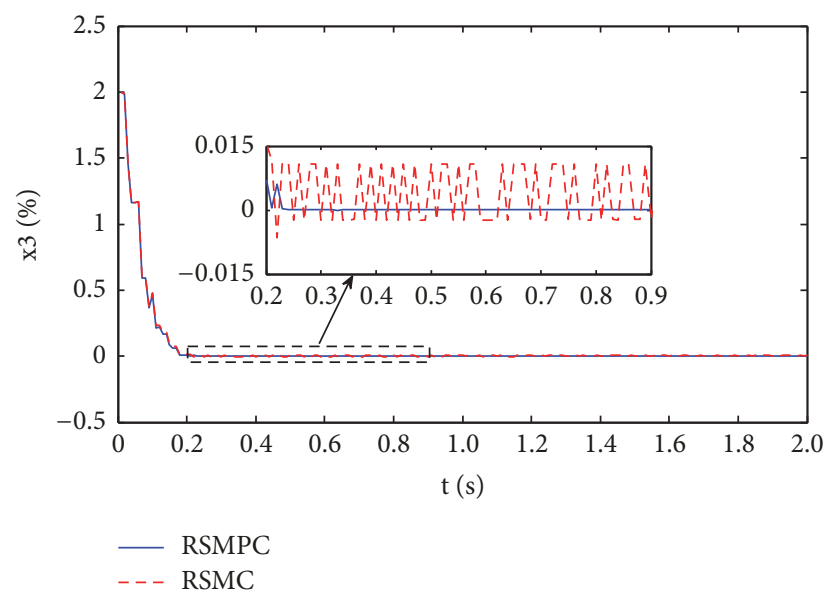

(c) Response curves of system state $x_{3}$

FIGURE 3: Response curves of system states with different controllers.

However, when system states reach the sliding surface, for the proposed controller, since prediction errors are obtained and analyzed to provide the optimal control inputs in every step, chattering can be suppressed to the maximum degree. But for the reaching law based sliding mode controller, there is no closed-loop feedback of errors or the optimization of control inputs, which makes it impossible to provide an ideal static performance.

Then, to verify the robustness of the proposed controller, another controller is introduced as comparison, whose 


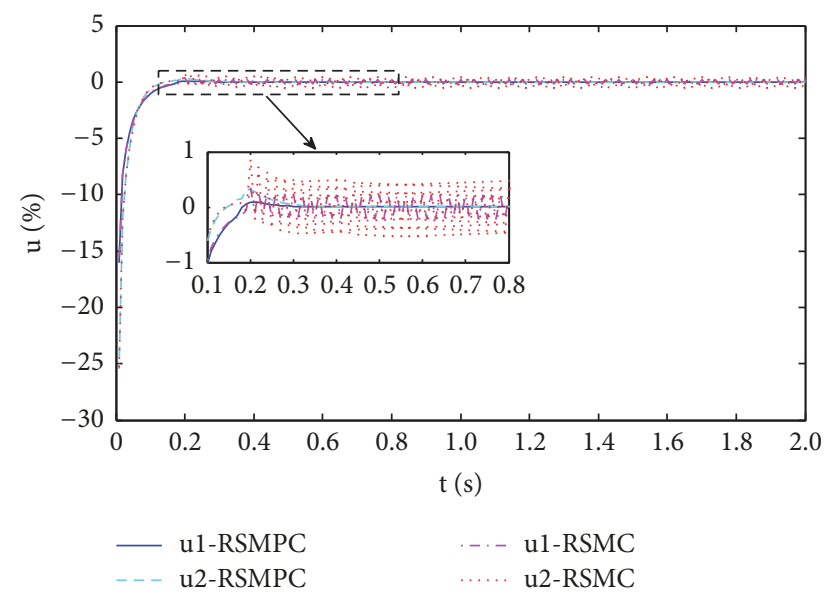

FIGURE 4: Evolution of sliding surfaces with different controllers.

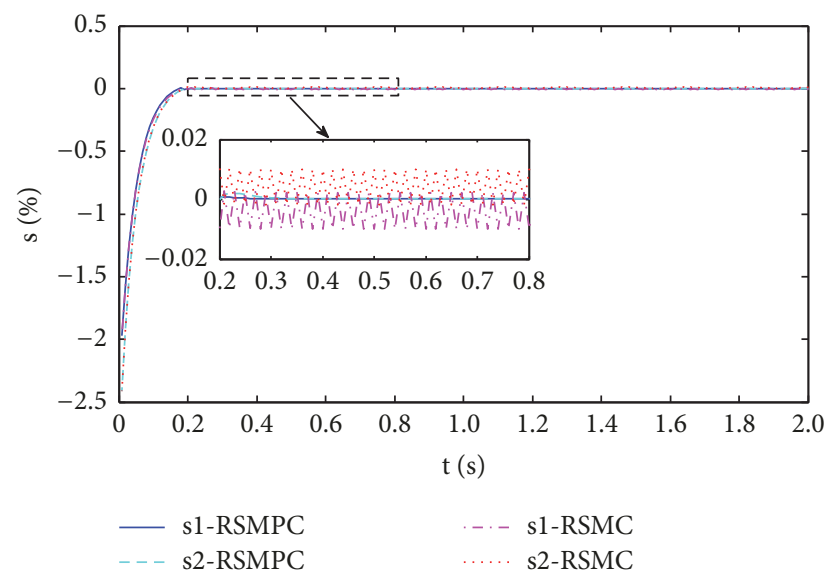

FIGURE 5: Evolution of control input with different controllers.

sliding surface parameter is not obtained from Theorem 5 but based on pole placement method [20], but it shares the same predictive sliding mode control strategy with the proposed controller. For the reduced system (8), define the target pole as $\lambda_{g}=-0.2$, then the sliding surface parameter can be obtained as

$$
\boldsymbol{G}_{\mathrm{p}}=\left[\begin{array}{lll}
9.5107 & 0.1 & 0.5 \\
7.6551 & 0.2 & 0.4
\end{array}\right]
$$

Here the controller whose sliding surface parameter is obtained based on pole placement method is denoted as PSMPC.

The disturbance condition of the previous example is $N_{i}=0.05$. Then the following four disturbance conditions are considered and the corresponding response curves of system state $x_{1}$ with the above three controllers (RSMPC, RSMC, PSMC) are shown in Figures 6-9.

$$
\begin{array}{ll}
\text { condition1: } & N_{1}=\operatorname{diag}(0.10,0.10,0.10) \\
\text { condition2: } & N_{2}=\operatorname{diag}(0.25,0.25,0.25)
\end{array}
$$

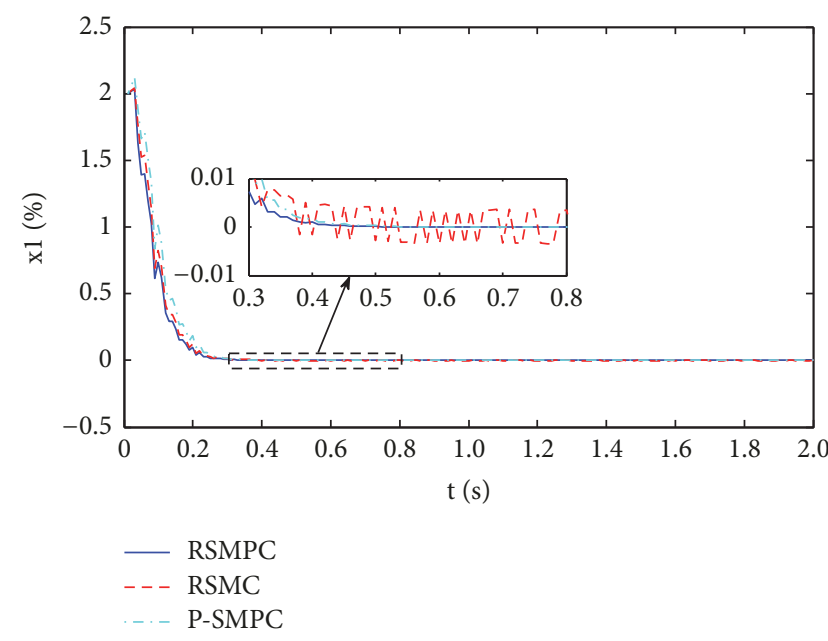

FIGURE 6: Response curves of system state $x_{1}$ under disturbance condition 1.

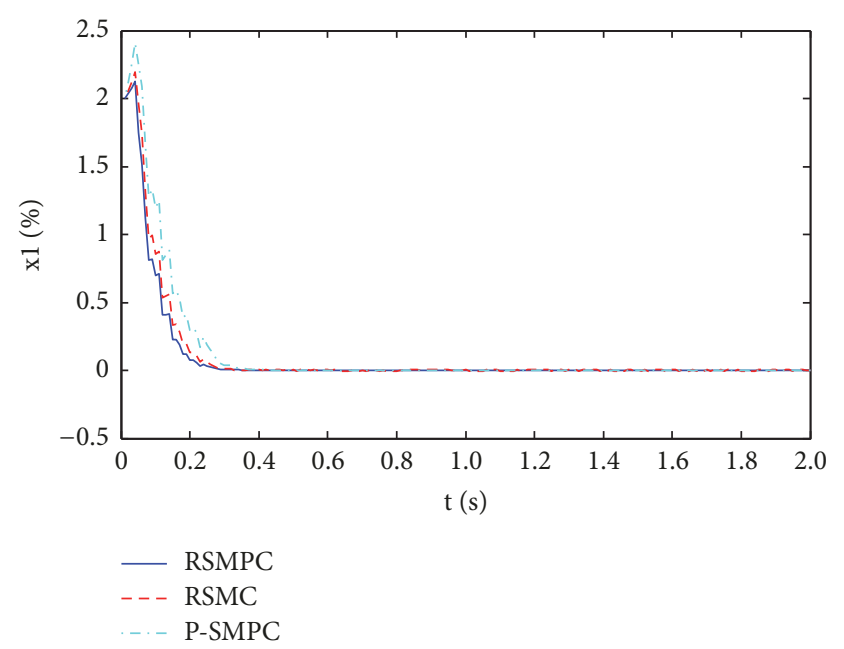

FIGURE 7: Response curves of system state $x_{1}$ under disturbance condition 2 .

$$
\begin{array}{ll}
\text { condition3: } & \boldsymbol{N}_{3}=\operatorname{diag}(0.5,0.5,0.5) \\
\text { condition4: } & \boldsymbol{N}_{4}=\operatorname{diag}(1.0,1.0,1.0)
\end{array}
$$

It can be seen from Figures 6-8 that, for the previous three disturbance conditions, all controllers can guarantee the stability of the system and it can be seen from Figure 6 that when the intensity of disturbance is at a low level, PSMPC also shows good static performance because of the adoption of predictive control strategy. However, when the intensity of disturbance grows, the dynamic performance of all controllers degrades but it is obvious that RSMC and PSMPC show much greater degradation, especially P-SMPC since robustness is not considered when designing its sliding surface parameter. Then when the intensity of disturbance moves to condition 4 with $N_{i}=1.0$, it can be seen from Figure 9 that RSMC and the proposed controller can still guarantee the stability of the system while P-SMPC fails to 


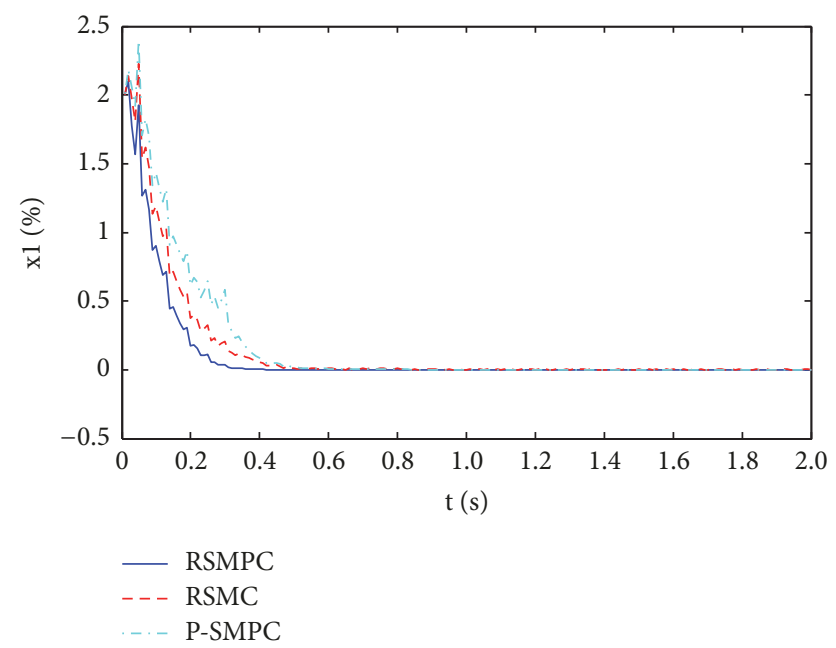

Figure 8: Response curves of system state $x_{1}$ under disturbance condition 3 .

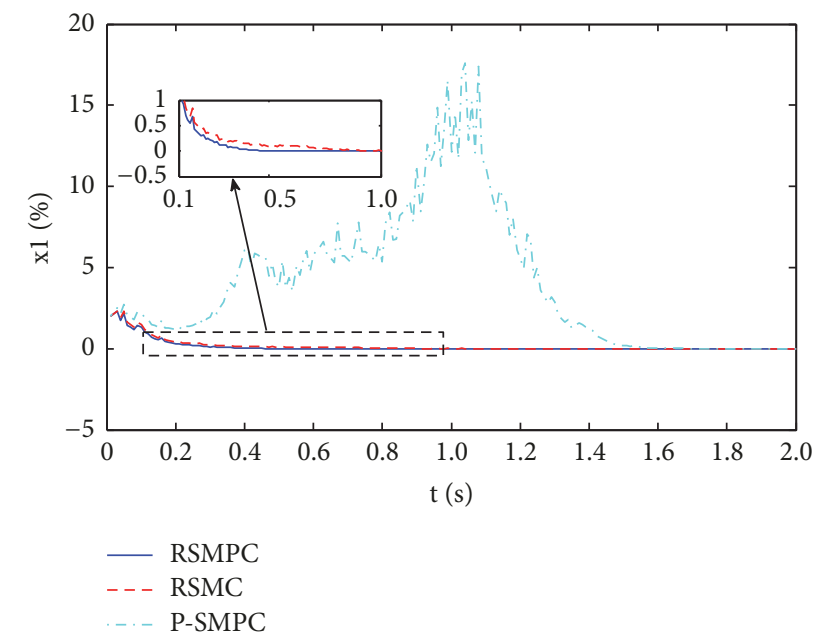

Figure 9: Response curves of system state $x_{1}$ under disturbance condition 4 .

make it. Therefore the robustness of the proposed sliding surface and the controller can thus be verified.

\section{Conclusion}

In this paper, a robust sliding mode predictive controller has been designed for a certain networked control system with random time delay, mismatched parametric uncertainty, and external disturbances. A global sliding surface has been designed which takes into account system robustness to mismatched uncertainties and the sufficient condition has been given in the form of matrix inequality (LMI) to guarantee system stability and robustness to uncertainties. Modified reaching law has been introduced as reference trajectory and strategies of feedback compensation and rolling optimization have been designed for the sliding mode predictive controller. Simulation results indicate that the proposed controller is advanced in both chattering suppression and robustness to uncertainties.

At last, it is necessary to talk about the deficiencies of the proposed method. Since system nominal model is adopted for the sliding mode predictive controller, high modeling accuracy is required, which may result in certain conservativeness. In our future work, uncertainties of modeling errors and parameter perturbations will be further considered and the model-based control strategy will be improved to reduce conservativeness.

\section{Data Availability}

The data used to support the findings of this study are included within the article.

\section{Conflicts of Interest}

The authors declare that they have no conflicts of interest.

\section{Acknowledgments}

This work was supported by the National Natural Science Foundation of China (51476187, 51606219, and 51506221).

\section{References}

[1] R. K. Belapurkar, R. K. Yedavalli, and B. Moslehi, "Stability of fiber optic networked decentralized distributed engine control under time delays," in Proceedings of the 45th AIAA/ ASME/SAE/ASEE Joint Propulsion Conference and Exhibit, USA, August 2009.

[2] W. Wu, "Fault-tolerant control of uncertain non-linear networked control systems with time-varying delay, packet dropout and packet disordering," IET Control Theory \& Applications, vol. 11, no. 7, pp. 973-984, 2017.

[3] B. Li, J.-F. Wu, and L. Huang, "Improved $\mathrm{H} \propto$ control for networked control systems with network-induced delay and packet dropout," Journal of Central South University, vol. 23, no. 5, pp. 1215-1223, 2016.

[4] M. A. Khanesar, O. Kaynak, S. Yin, and H. Gao, "Adaptive indirect fuzzy sliding mode controller for networked control systems subject to time-varying network-induced time delay," IEEE Transactions on Fuzzy Systems, vol. 23, no. 1, pp. 205-214, 2015.

[5] Z.-D. Tian, X.-W. Gao, B.-L. Gong, and T. Shi, “Time-delay compensation method for networked control system based on time-delay prediction and implicit PIGPC," International Journal of Automation and Computing, vol. 12, no. 6, pp. 648656, 2015.

[6] A. Selivanov and E. Fridman, "Predictor-based networked control under uncertain transmission delays," Automatica, vol. 70, pp. 101-108, 2016.

[7] T. Jia, Y. Niu, and Y. Zou, "Sliding mode control for stochastic systems subject to packet losses," Information Sciences, vol. 217, pp. 117-126, 2012.

[8] J. J. Moghaddam, M. H. Farahani, and N. Amanifard, "A neural network-based sliding-mode control for rotating stall and surge in axial compressors," Applied Soft Computing, vol. 11, no. 1, pp. 1036-1043, 2011. 
[9] A. K. Khatri, J. Singh, and N. K. Sinha, "Aircraft maneuver design using bifurcation analysis and sliding mode control techniques," Journal of Guidance, Control, and Dynamics, vol. 35, no. 5, pp. 1435-1449, 2012.

[10] A. Nizar, B. M. Houda, and N. A. Said, "A new sliding function for discrete predictive sliding mode control of time delay systems," International Journal of Automation and Computing, vol. 10, no. 4, pp. 288-295, 2013.

[11] D. Sonawane, D. Ingole, and V. Naik, "FPGA implementation of linear model predictive controller for real-time position control of DC motor," International Journal of Circuits and Architecture Design, vol. 1, no. 4, p. 281, 2015.

[12] J. Zhou, L. Fiorentini, and M. Canova, "Model-based optimisation and predictive control of a turbocharged diesel engine with variable geometry compressor," International Journal of Powertrains, vol. 5, no. 2, p. 167, 2016.

[13] L. Ben Hamouda, M. Ayadi, and N. Langlois, "Takagi-Sugeno fuzzy observer and predictive control for a diesel engine air path," International Journal of Automation and Control Engineering, vol. 9, no. 4, pp. 283-302, 2015.

[14] W. Garcia-Gabin, D. Zambrano, and E. F. Camacho, "Sliding mode predictive control of a solar air conditioning plant," Control Engineering Practice, vol. 17, no. 6, pp. 652-663, 2009.

[15] B. Kim, G. N. Washington, and H. S. Yoon, "Hysteresis-reduced dynamic displacement control of piezoceramic stack actuators using model predictive sliding mode control," Smart Materials \& Structures, vol. 21, no. 21, pp. 55018-55030, 2012.

[16] D. M. Raimondo, M. Rubagotti, C. N. Jones, L. Magni, A. Ferrara, and M. Morari, "Multirate sliding mode disturbance compensation for model predictive control," International Journal of Robust and Nonlinear Control, vol. 25, no. 16, pp. 29843003, 2015.

[17] F. Da, "Sliding mode predictive control for long delay time systems," Physics Letters A, vol. 348, no. 3-6, pp. 228-232, 2006.

[18] H. Alwi, C. Edwards, and C. P. Tan, "Sliding mode estimation schemes for incipient sensor faults," Automatica, vol. 45, no. 7, pp. 1679-1685, 2009.

[19] J. Q. Candela, A. Girard, and J. Larsen, "Propagation of uncertainty in Bayesian kernel models-application to multiplestep ahead forecasting," in Proceeding of IEEE International Conference on Acoustics, Speech, and Signal Processing, pp. 701704, Lyngby, 2003.

[20] W. B. Gao, Theory and Designing Method of Variable Structure Control, Science Press, Beijing, 1996.

[21] L. Xiao, H. Su, X. Zhang, and J. Chu, "A new variable structure control algorithm based on general sliding mode prediction," in Proceedings of the 2006 th World Congress on Intelligent Control and Automation, pp. 2141-2145, Dalian, China, 2006.

[22] Z. He, M. Wang, and S. Liu, "Discrete sliding mode prediction control of uncertain switched systems," Journal of Systems Engineering and Electronics, vol. 20, no. 5, pp. 1065-1071, 2009. 


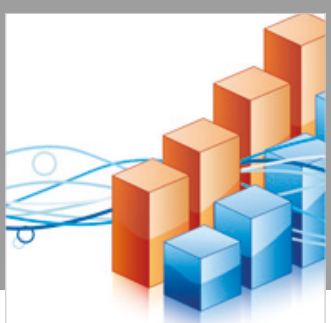

Advances in

Operations Research

\section{-n-m}
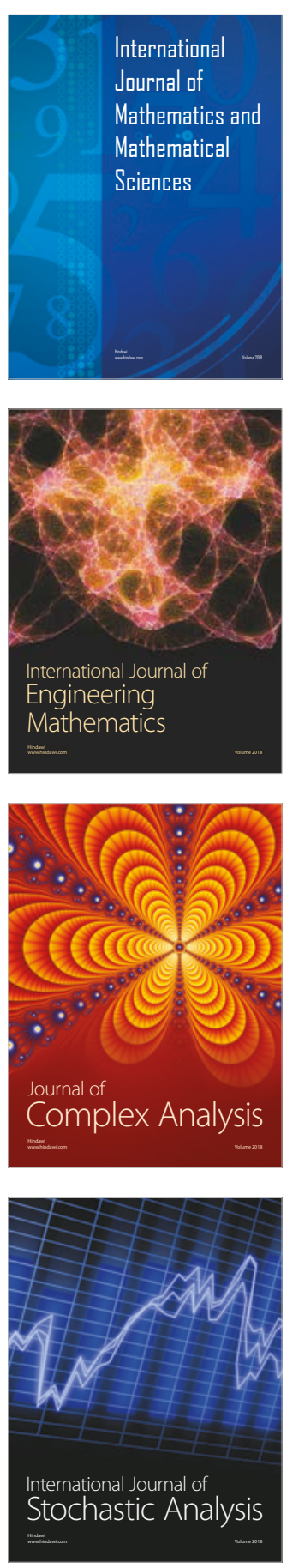
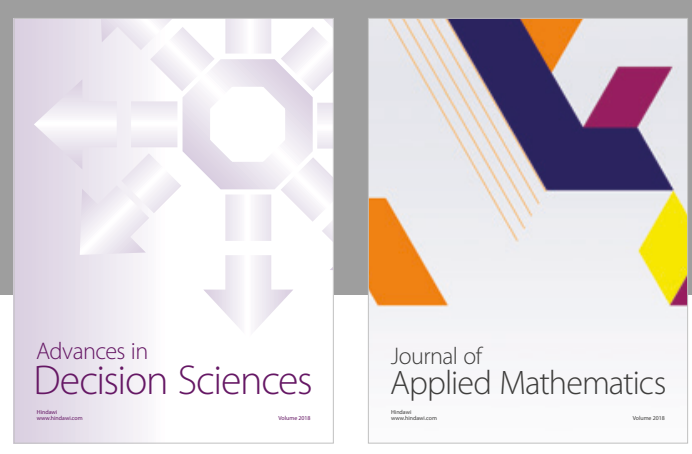

Journal of

Applied Mathematics
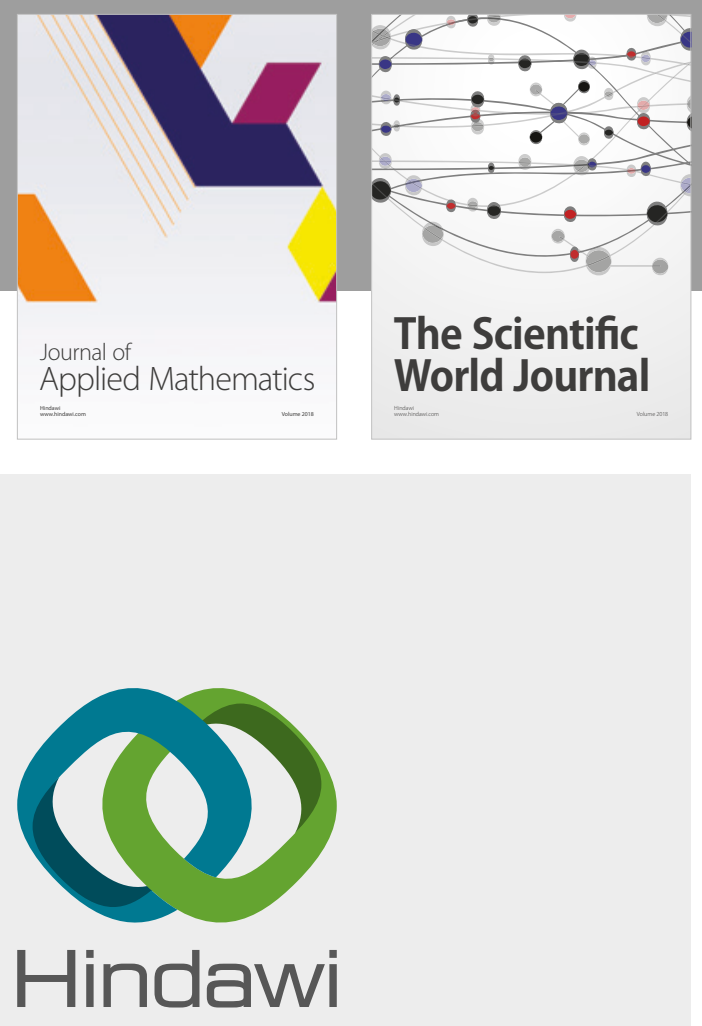

Submit your manuscripts at

www.hindawi.com

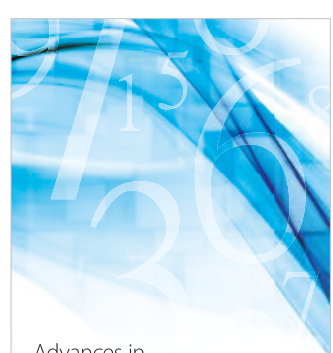

Advances in
Numerical Analysis
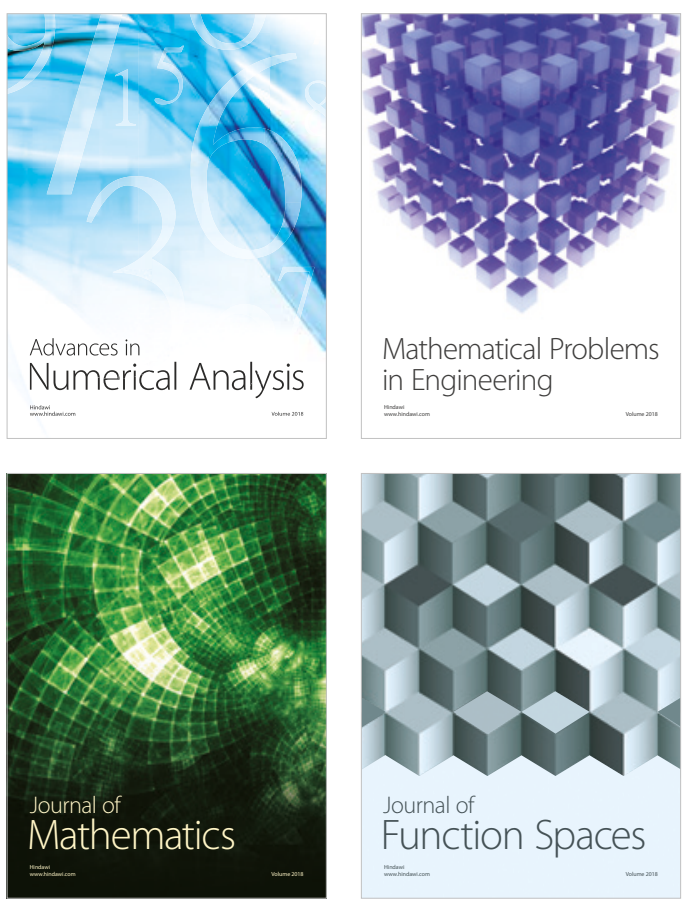

Mathematical Problems in Engineering

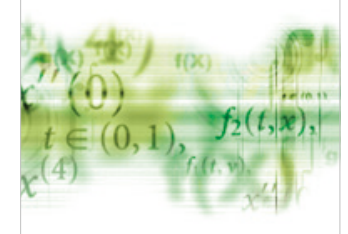

International Journal of

Differential Equations

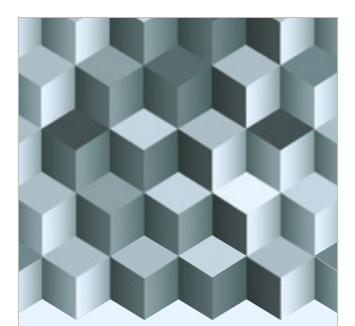

Journal of

Function Spaces

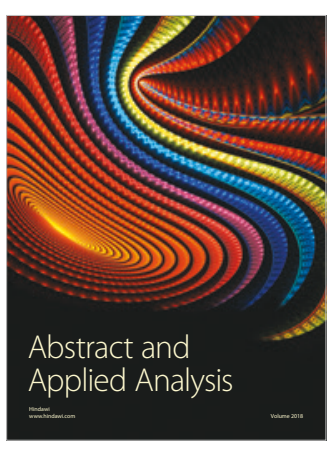

The Scientific

World Journal

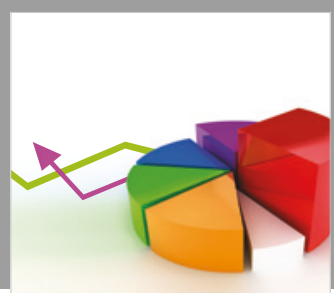

Journal of

Probability and Statistics
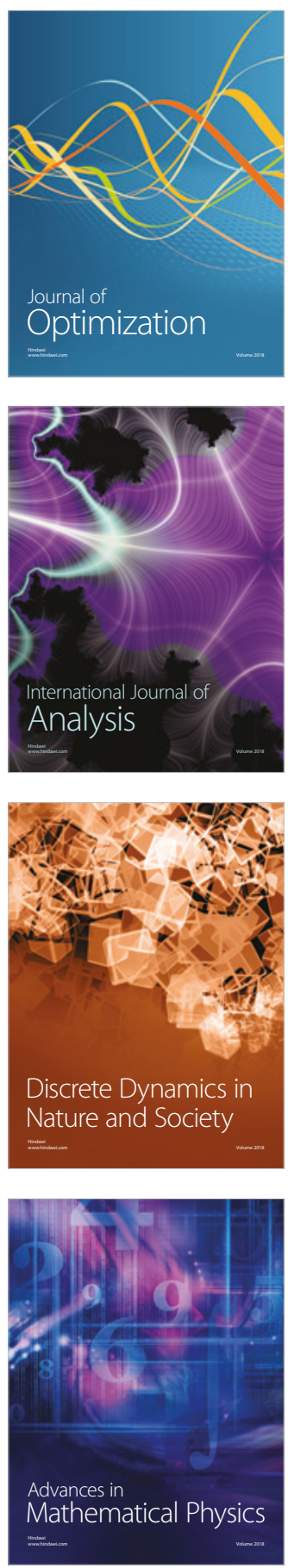